\title{
High Performance Organic Field-Effect Transistors using Ambient Deposition of Tetracene Single Crystals
}

Logan A. Morrison ${ }^{\mathrm{a}}$, Dane Stanfield ${ }^{\mathrm{b}}$, Michael Jenkins ${ }^{\mathrm{b}}$, Alexandr A. Baronov ${ }^{\mathrm{b}}$, David L. Patrick $^{\mathrm{b}}$, Janelle M. Leger ${ }^{\mathrm{a}, \mathrm{b}}$,*

${ }^{a}$ Department of Physics and Astronomy, Western Washington University, 516 High St, Bellingham, WA, 98225-9164, United States

${ }^{\mathrm{b}}$ Department of Chemistry, Western Washington University, 516 High St, Bellingham, WA, 98225-9150, United States

*Contact Author Email: Janelle.leger@wwu.edu

Keywords: Organic-Vapor-Liquid-Solid Deposition; OFET; Tetracene; Ambient Crystal Growth; Organic Electronics

\begin{abstract}
Organic molecular crystals (OMCs) are of significant interest due to their potential use in transistors, photovoltaic devices, light emitting diodes, and other applications. However, conventional vacuum-based methods of growing crystalline OMC films are costly and provide limited control over crystal growth. In this study, we present a new method for preparing high performance single-crystal tetracene field-effect transistors under near-ambient conditions using organic vapor-liquid-solid (OVLS) deposition. We find that the mobility of OVLS-grown tetracene is comparable to high quality crystalline films prepared by physical vapor deposition. These results establish OVLS deposition as a relatively low cost, low substrate temperature, and ambient pressure method for growing high quality OMC films for device applications.
\end{abstract}




\section{Introduction}

Over the past 30 years, organic molecular crystals (OMCs) have received increasing attention due to their interesting and potentially useful electrical, optical and magnetic properties. ${ }^{[1]}$ Relatively high charge carrier mobilities as compared to their thin-film counterparts make organic single-crystals a promising choice for device applications such as photovoltaic devices and organic-field effect transistors (OFETs). ${ }^{[2-4]}$ The outstanding device performance ${ }^{[5-11]}$ of OFETs made using OMCs makes them attractive for electronic applications such as active matrix displays and sensor arrays. However, for practical applications, it is necessary to grow OMCs using a low cost deposition method compatible with high-throughput, large area deposition. While many solution-processing methods are available for fabricating OMC layers ${ }^{[12]}$, most require a fairly high degree of solubility for the active material. Because many OMC compounds have poor solubility, crystalline films are often grown instead using methods such as physical vapor transport or physical vapor deposition (PVD). ${ }^{[13]}$ These methods, while applicable to a broad class of materials, are relatively expensive to perform, tend to have low throughput, and provide limited control over crystal growth habit, orientation and size. ${ }^{[14]}$ In order for OMCs to become practical for device applications, an inexpensive, high throughput method compatible with a broad class of materials and providing control over crystal morphology and orientation is highly desirable.

Here we employ a hybrid deposition technique known as organic-vapor-liquid-solid (OVLS) deposition to prepare single-crystal OMC films at near-ambient conditions. ${ }^{[15-18]}$ OVLS deposition is performed by sublimating an organic precursor into a stream of an inert carrier gas, which carries it to a substrate coated by a thin liquid solvent layer. Arriving monomers impinge upon and dissolve into the solvent, raising the concentration until crystals form in a quasi-2 dimensional layer by a burst nucleation mechanism ${ }^{[18]}$. The method is an ambient-pressure, all-organic analog to the VLS technique of crystal growth introduced in the 1960's by Wagner and Ellis for inorganic materials in liquid metal alloy droplets, ${ }^{[19,20]}$ but 
employs an organic solvent combined with an organic precursor delivered via the vapor phase. The OVLS growth method has been previously used to prepare films of tetracene, ${ }^{[16-18]}$ pentacene,${ }^{[21]}$ rubrene, ${ }^{[22,23]}$ buckminsterfullerene, ${ }^{[24]}$ and other molecular systems, ${ }^{[25-29]}$ demonstrating its applicability for a broad class of OMC materials covering a wide range of solubilities. Additionally, OVLS deposition can be used to control crystal morphology though interactions between the solvent and organic precursor, and by using a liquid crystal solvent and/or patterning, it may also be possible to fabricate arrays of oriented crystals to maximize charge carrier mobility and performance for devices such as FETs. ${ }^{[17]}$

Despite its promise as an applications-relevant OMC film deposition method, to date no demonstrations of the use of OVLS growth to prepare films suitable for device applications have been reported. In this paper, we present a simple method for preparing device-quality OVLS-deposited OMC films. The resulting films are used in the fabrication of OFETs in order to assess how their electrical properties compare to crystalline films prepared by conventional PVD methods. We find that OVLS-grown tetracene films exhibit performance comparable to the best literature values, demonstrating formation of high-quality crystalline films under near-ambient conditions.

\section{Experimental Section}

Field-effect transistors were fabricated under inert atmosphere. Heavily p-doped silicon was used at the gate electrode with a $300 \mathrm{~nm}$ thermally grown $\mathrm{SiO}_{2}$ layer as the gate dielectric. The silicon was first ozone cleaned to improve wettability of the OVLS growth solvent. The growth solvent layer thickness was measured by optical interferometry. Details of the OVLS deposition procedure for the active layer are given in section 3. X-ray diffractometry of the resulting films was performed with a Panalytical Xpert diffractometer using a $\mathrm{Cu} \mathrm{K} \alpha$ source $(\lambda=0.154 \mathrm{~nm})$. 
Thermal evaporation of gold $(\sim 40 \mathrm{~nm})$ as the source and drain electrodes at $2 \AA / \mathrm{s}$ was performed following overnight pump down under high vacuum $\left(10^{-7}\right.$ Torr $)$ using a high density shadow mask (Ossilla Ltd.). After electrode deposition, devices were annealed at 100 ${ }^{\circ} \mathrm{C}$ under nitrogen for a further 30 minutes to ensure good contact between electrodes and tetracene crystals. Silver epoxy was painted on the side of the silicon substrate in order to make contact to the gate electrode. Device testing was carried out in a nitrogen glovebox with $<0.1 \mathrm{ppm}$ of oxygen at room temperature to avoid oxidation of tetracene. Electrical measurements were taken using two Keithley 2400 SourceMeters.

\section{Results and Discussion}

Field-effect transistors were fabricated in a top-contact configuration. Following preparation of the $\mathrm{Si} / \mathrm{SiO}_{2}$ substrate, a 1100 um-thick layer of 2,6,10,15,19,23hexamethyltetracosane (squalane) was spin-coated on the substrate, serving as the growth solvent. Squalane was chosen for its low vapor pressure, low cost, low toxicity, and favorable wetting of tetracene crystals. Tetracene films were deposited using a custom OVLS deposition chamber (Figure 1). Tetracene vapor generated in a crucible held at $250{ }^{\circ} \mathrm{C}$ was swept into a stream of heated $\mathrm{N}_{2}$, with flow directed through a nozzle onto the Si substrate (flow rate 0.1 standard $\mathrm{L} \mathrm{min}^{-1}$ ). The nozzle has a slightly tapered constriction at its end, resulting in pluglike flow impinging on the substrate with axisymmetric stagnation point geometry. This produces nearly uniform deposition over an area equal to the size of the nozzle, $\sim 1 \mathrm{~cm}^{2}$. A retractable shutter is used to start and stop the deposition. The typical deposition time was 90 min. Further details of the OVLS deposition method, including treatment of the flow dynamics, are given in Ref. 15.

Tetracene carried to the substrate by the impinging flow dissolves in the squalane layer, raising the concentration until a critical supersaturation is reached, causing crystals to nucleate. Subsequent growth of these crystals partially depletes the solution of monomers, halting the 
formation of new crystals, but permitting growth of existing crystals fed by the ongoing vapor-phase flux. The result is a burst of nucleation, followed by growth, with crystals fully submerged in the solvent layer. ${ }^{[18]}$ Crystals grew as thin platelets with prismatic morphologies, ranging in size from $100-500 \mu \mathrm{m}$ and having a typical thickness determined by atomic force microscopy of $150-250 \mathrm{~nm}$ (Figure 2(a,b)). X-ray diffractometry performed on these samples agreed with the previously reported bulk crystal structure of tetracene, ${ }^{[30,31]}$ and showed crystals possessing random in-plane, but uniform out-of-plane alignment, with the $a b$-crystal plane oriented parallel to the substrate (Figure 2(c)). High resolution polarized optical microscopy indicated the majority were single-crystals, undergoing uniform extinction upon sample rotation when viewed between crossed polarizers (Figure 2(d)-(f)). Film growth was halted at a coverage well below the point where crystals began to coalesce, producing large, widely-separated crystals suitable for single-crystal device measurements. More detailed descriptions of the growth kinetics and coverage-dependent morphological evolution of these films is given in Refs. 15 and 18.

Following tetracene deposition, removal of the liquid solvent layer is necessary for device fabrication. This step must be performed carefully to avoid degradation of the film. We found that incomplete removal of the squalane layer led to significant threshold voltage shifts, decaying output characteristics and low charge carrier mobilities as illustrated in Figure S1 in the supplementary materials. The observed threshold voltage shifts and decaying output characteristics are similar to those previously observed and attributed to charge traps accumulating at the dielectric/semiconductor interface ${ }^{[32,33]}$. We found squalane could be effectively removed with no measureable changes to the tetracene film by heating samples to $55{ }^{\circ} \mathrm{C}$ at $10^{-4}$ Torr for 48 hours, followed by annealing at $100^{\circ} \mathrm{C}$ for 30 minutes in a nitrogen glovebox.

OFETs with a channel length of $10 \mu \mathrm{m}$ and a channel width of $1000 \mu \mathrm{m}$ were constructed with gold source and drain electrodes as described in section 2. A schematic of the OFET 
architecture and an optical image of the completed device are shown in Figure 3. Output scans were acquired between $\mathrm{V}_{\mathrm{GS}}=0 \mathrm{~V}$ to $-100 \mathrm{~V}$ in $20 \mathrm{~V}$ increments and $\mathrm{V}_{\mathrm{DS}}$ was stepped from 0 $\mathrm{V}$ to $-100 \mathrm{~V}$ in $1 \mathrm{~V}$ increments. Linear transfer scans were taken at $\mathrm{V}_{\mathrm{DS}}=-10 \mathrm{~V}$ while $\mathrm{V}_{\mathrm{GS}}$ was stepped from $0 \mathrm{~V}$ to $-100 \mathrm{~V}$ in $1 \mathrm{~V}$ increments. Output and linear transfer characteristic scans for a typical device are shown in Figures 4A and 4B. The linear charge carrier mobility is calculated using the slope of the drain current vs. gate voltage using ${ }^{[34]}$

$$
I_{D S}=\frac{W C_{o x}}{2 L} \mu_{l i n}\left(V_{G S}-V_{T}\right) V_{D S}
$$

and the saturation mobility was calculated using the slope of the square root of the drain current vs. the gate voltage using ${ }^{[35]}$

$$
I_{D S}=\frac{W C_{o x}}{2 L} \mu_{s a t}\left(V_{G S}-V_{T}\right)^{2}
$$

where $I_{S D}$ is the drain current, $\mu_{\text {lin }}$ and $\mu_{s a t}$ are the linear and saturation charge carrier mobilities respectively, $V_{G S}$ is the gate voltage, $V_{T}$ is the threshold voltage and $V_{D S}$ is the drain voltage, $\mathrm{L}$ is the channel length and $W$ is the channel width. The actual channel width was determined by measuring the crystal width using an Olympus optical microscope. $C_{o x}=11.8$ $\mathrm{nF} \mathrm{cm}{ }^{-2}$ is the capacitance per unit area of the $\mathrm{SiO}_{2}$ layer calculated using $C_{o x}=\frac{\epsilon_{r} \epsilon_{0}}{d}$, where $\varepsilon_{\mathrm{r}}$ is the dielectric constant of $\mathrm{SiO}_{2}, 3.9, \varepsilon_{0}$ is the permittivity of free space and $\mathrm{d}$ is the thickness of the $\mathrm{SiO}_{2}$ layer $(300 \mathrm{~nm})$.

For the device shown in Figure 4, the linear charge carrier mobility was found to be $0.10 \mathrm{~cm}^{2} / \mathrm{Vs}$ with an on/off ratio of $3.5 \times 10^{5}$ and a threshold voltage of $-37.34 \mathrm{~V}$. Saturation scans were taken at $V_{D S}=-80 \mathrm{~V}$. The saturation mobility was found to be $0.30 \mathrm{~cm}^{2} \mathrm{~V}^{-1} \mathrm{~s}^{-1}$. In addition, an analysis of the contact resistance in this device is presented in the supplemental 
information (Figure S2), calculated using the method outlined in [38]. In general, the linear and saturation mobilities calculated over a total of 11 working devices was $(0.10 \pm 0.06)$ $\mathrm{cm}^{2} / \mathrm{Vs}$ and $(0.15 \pm 0.07) \mathrm{cm}^{2} / \mathrm{Vs}$, respectively. These mobilities agree well with those measured in devices based on tetracene single crystals grown by physical vapor deposition or solution processing on $\mathrm{SiO}_{2},{ }^{[36,37]}$ evidence of the high quality of the crystals formed in these films.

\section{Conclusion}

In this work, high performance single-crystal OFETs prepared under near-ambient conditions by organic-vapor-liquid-solvent (OVLS) deposition were demonstrated for the first time. We show that squalane serves as a low-cost, low-toxicity solvent for OVLS and can be readily removed by a simple low-temperature process, enabling growth of large, high-quality crystals suitable for device integration. Linear and saturation charge carrier mobilities of OVLS-deposited tetracene are found to be comparable to those reported using single-crystals grown by conventional vacuum methods. These results establish OVLS growth as a route to relatively low-cost preparation of applications-ready OMC films.

\section{Acknowledgements}

This work was supported by the National Science Foundation under Grants No. DMR1508591, DMR-1207338, and DMR 1057209.

\section{References}

[1] G. R. Desiraju, The Design of Organic Solids, (Elsevier, NY, 1989)

[2] R. F. Service, Science, 278, 383 (1997)

[3] F. Garnier, R. Hajlaoui, A. Yassar and P. Srivastava, Science, 265, 1684 (1994)

[4] C. J. Drury, C. M. Mutsaers, C. M. Hart, M. Matters and D. M. Leeuw, Appl. Phys. Lett. 73, 108 (1998) 
[5] A. L. Briseno, J. Aizenberg, Y. Han, R. A. Penkala, H. Moon, A. J. Lovinger, C. Kloc and Z. Bao, J. Am. Chem. Soc. 127, 12164 (2005)

[6] H. E. A. Huitema, G. H. Gelinck, J. B. P. H. Van Der Putten, K. E. Kuijk, C. M. Hart, E. Cantatore, P. T. Herwig, A. J. J. M. Van Breemen and D. M. De Leeuw, Nature 414, $599(2001)$

[7] H. Moon, R. Zeis, E.J. Borkent, C. Besnard, A. J. Lovinger, T. Siegrist, C. Kloc and Z. Bao, J. Am. Chem. Soc. 126, 15322 (2004)

[8] V. Podzorov, E. Menard, A. Borissov, V. Kiryukhin, J. A. Rogers and M. E. Gershenson, Phys. Rev. Lett. 93, 086602 (2004)

[9] M. Pope, E. Charles and C. E. Swenberg, Electronic Processes in Organic Crystals and Polymers, $2^{\text {nd }}$ ed. (Oxford University Press, Oxford, 1999)

[10] V. C. Sundar, J. Zaumseil, V. Podzorov, E. Menard, R. L. Willett, T. Someya, M. E. Gershenson and J. A. Rogers, Science, 303, 1644, (2004)

[11] R. Zeis, C. Besnard, T. Siegrist, C. Schlockermann, X. Chi and C. Kloc, Chem. Mater., 18, 244 (2006)

[12] L. Shaw and Z. Bao, Isr. J. Chem. 54, 496 (2014)

[13] A. Afzali, C. D. Dimitrakopoulos and T. L. Breen, J. Am. Chem. Soc.124, 8812 (2002)

[14] S. R. Forrest, Chem. Rev. 97, 1793 (1997)

[15] D. W. Shaw, K. Bufkin, A. A. Baronov, B. L. Johnson and D. L. Patrick. J. Appl. Phys. 111, 074907 (2012)

[16] M. Voigt, S. Dorsfield, A. Volz and M. Sokolowski, Phys. Rev. Lett. 91, 026103 (2003)

[17] F. S. Wilkinson, R. F. Norwood, J. M. McLellan, L. R. Lawson and D. L. Patrick, J. Am. Chem. Soc. 128, 16468 (2006)

[18] A. Baronov, K. Bufkin, D. W. Shaw, B. L. Johnson, and D. L. Patrick, Phys. Chem. Chem. Phys. 17, 20846 (2015)

[19] R. S. Wagner and W. C. Ellis, Appl. Phys. Lett. 4, 89 (1964) 
[20] R. S. Wagner, Whisker Technology, Chap. 3, ed. A. P. Levitt (Wiley, New York, 1970).

[21] Y. Takeyama, S. Mantoku, S. Maruyama and Y. Matsumumoto, CrystEngComm 16, $684(2014)$

[22] S. Horike, Y. Koshiba, M. Misaki and K. Ishida, Jpn. J. Appl. Phys. 53, 05FT03 (2014)

[23] T. Shimada, Y. Ishi, K. Ueono and T. Hasegawa, J. Cryst. Growth, 311, 163 (2008)

[24] Y. Takeyama, S. Maruyama, H. Taniguchi, M. Itoh, K. Ueno and Y. Matsumoto, CrystEngComm, 14, 4939 (2012)

[25] X. Liu and M. Wuttig, Phys. Rev. B, 73, 033405 (2006)

[26] Y. Ishii, T. Shimada, N. Okazaki and T. Hasegawa, Langmuir, 23, 6864 (2007)

[27] T. Shimada, Y. Ishii, K. Ueno, N. Yoshimoto and T. Hasegawa, J. Cryst. Growth, $311,163(2008)$

[28] Y. Takeyama, S. Maruyama and Y. Matsumoto, Cryst. Growth Des. 11, 2273 (2011)

[29] Y. Takeyama, S. Ono and Y. Matsumoto, Appl. Phys. Lett. 101, 083303 (2012)

[30] J. M. Robertson, V. C. Sinclair and J. Trotter, Acta. Cryst., 14, 697 (1961)

[31] R. B. Campbell, J. M. Robertson and J. Trotter, Acta Cryst. 15, 289 (1962)

[32] S. G. J. Mathijssen, M. Kemerink, A. Sharma, M. Colle, P. A. Bobbert, R. A. J. Janssen, and D. M. de Leeuw, Adv. Mater. 20, 975 (2008)

[33] R. Häusermann and B. Batlogg, Appl. Phys. Lett. 99, 083303 (2011)

[34] G. Horowitz and P. Delannoy, J. Appl. Phys. 70, 469 (1991)

[35] S. M. Sze, Physics of Semiconductor Devices, (Wiley-Interscience, NY,1981)

[36] C. Chien, C. Lin, M. Watanabe, Y. Lin, T. Chao, T. Chiang, X. Huang, Y. Wen, C. Tu, C. Sun, and T. J. Chow, J. Mater. Chem. 22, 13070 (2012)

[37] R. W. I. de Boer, T. M. Klapwijk and A. F. Morpurgo, Appl. Phys. Lett. 83, 4345

(2003)

[38] D. J. Gundlach, L. Zhou, J. A. Nichols, T. N. Jackson, P. V. Necliudov, and M. S. Shur, J. Appl. Phys. 100, 024509 (2006). 


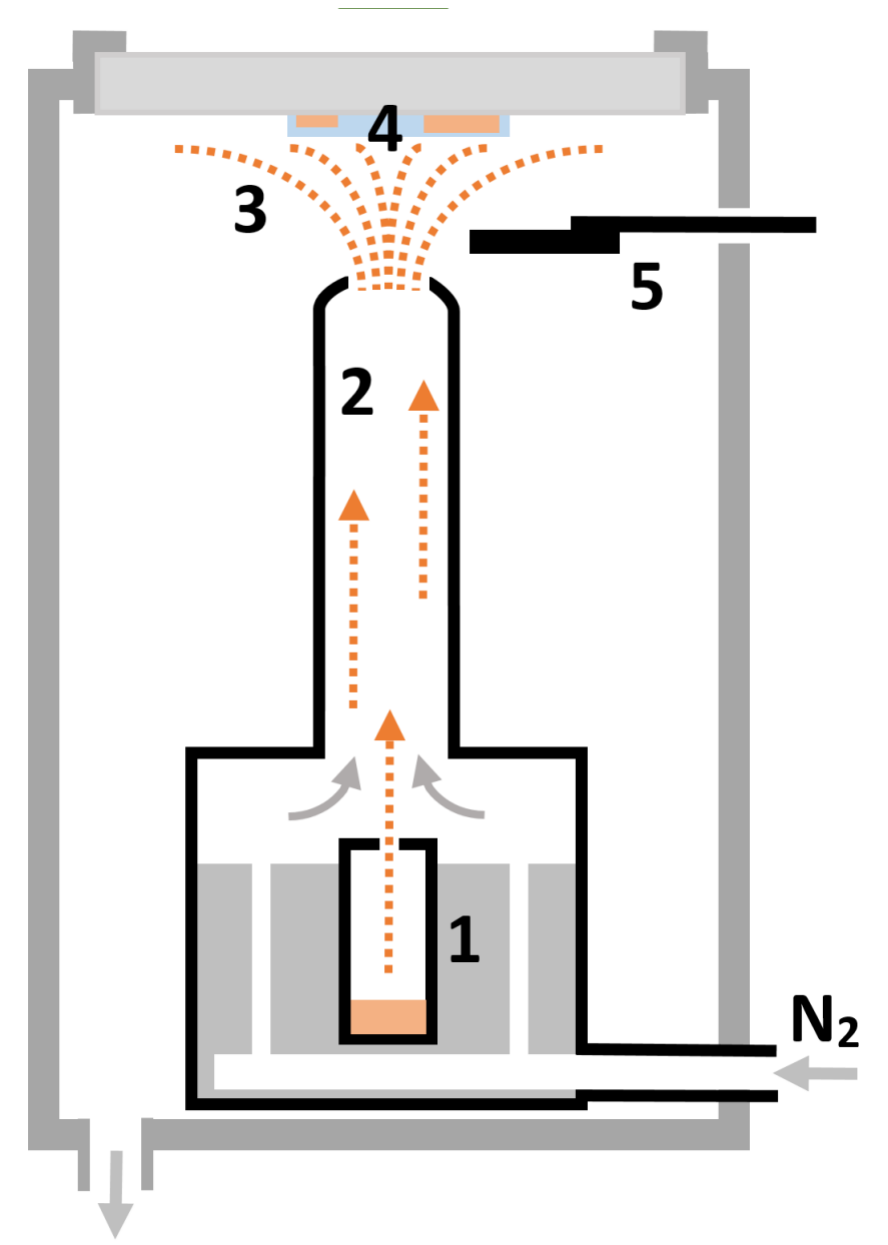

Figure 1. (A) Tetracene vapor generated in a heated crucible (1) is swept into a stream of heated $\mathrm{N}_{2}$ gas and carried up a tapered nozzle (2), impinging by axisymmetric stagnation point flow (3) on a squalane-coated $\mathrm{SiO}_{2} / \mathrm{Si}$ substrate (4). The steady flux of tetracene into the squalene solvent layer results in the formation of a finite number of micron-sized tetracene single-crystals via a burst nucleation mechanism. Deposition is controlled by a retractable shutter (5). 

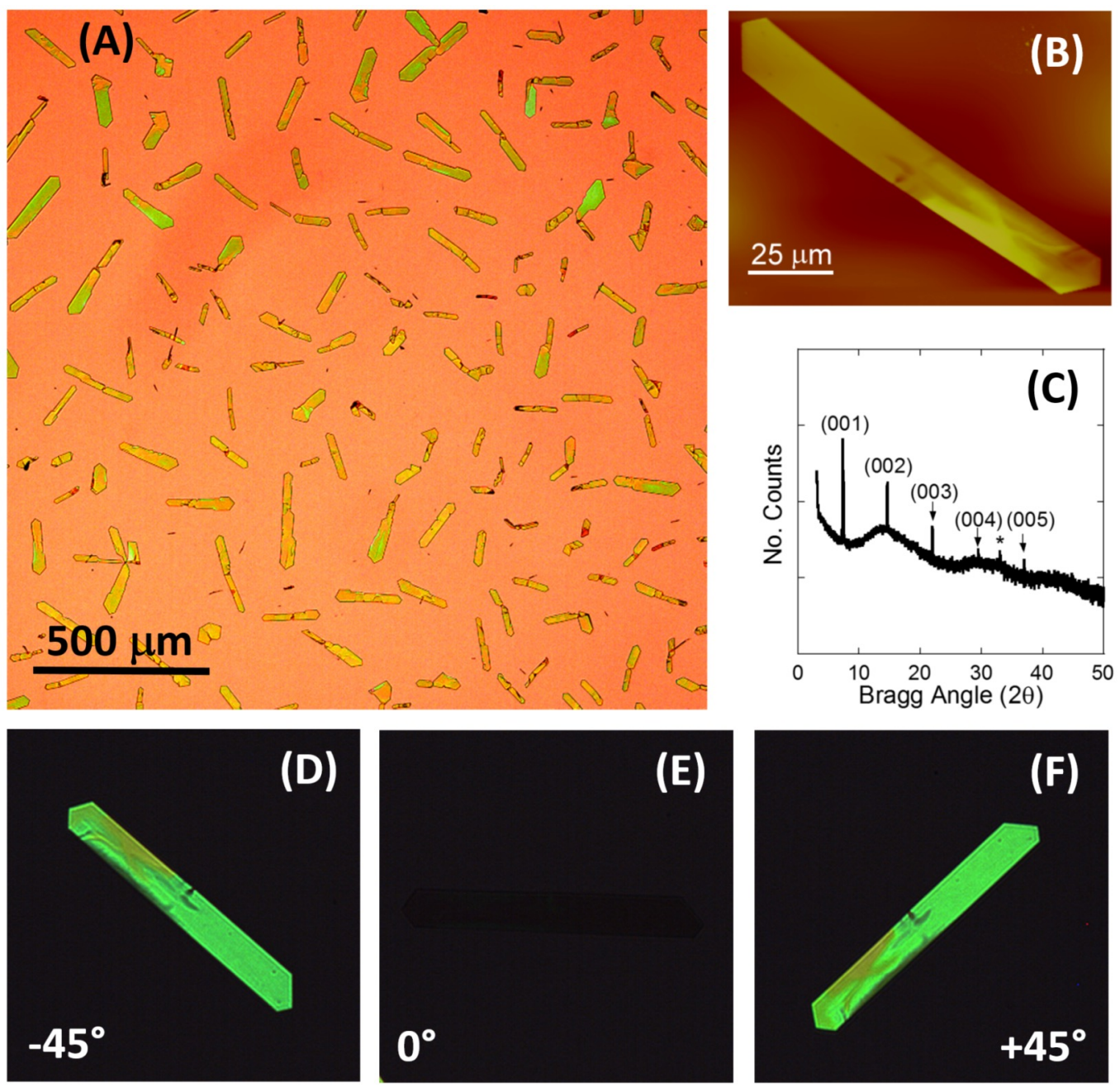

Figure 2. (A) Optical micrograph of an OVLS-grown tetracene film on $\mathrm{SiO}_{2}$. (B) Atomic force microscopy image of a representative crystal. Crystals ranged in thickness from $150-250 \mathrm{~nm}$. (C) Xray diffractogram after solvent removal. Reflections are observed exclusively from the $(00 l)$ family of planes, indicating the $a b$-crystallographic plane is parallel to the substrate. The peak labeled with an asterisk is from the Si substrate. (D-F) High resolution polarized optical micrographs of the crystal shown in part (B). Crystals undergo uniform extinction when rotated between crossed polarizers. 

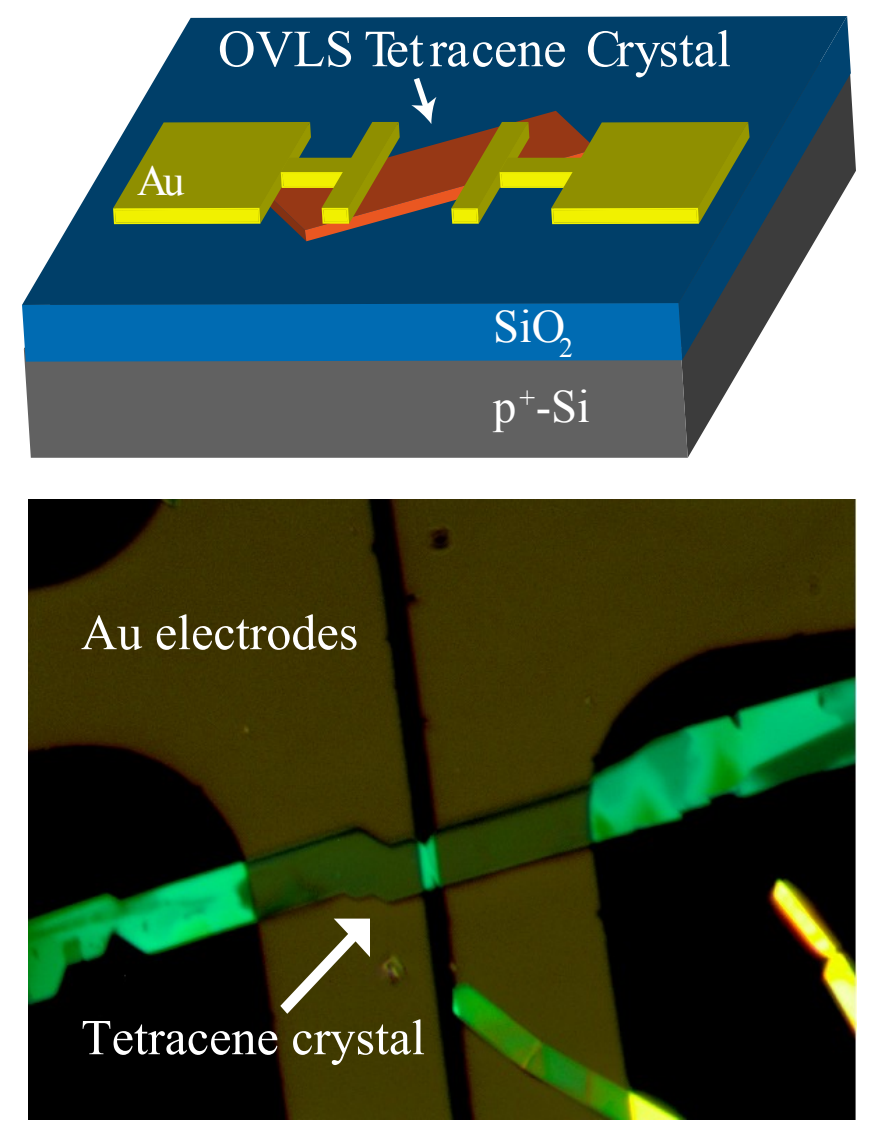

Figure 3. Schematic (above) and optical micrograph (below) of top-contact OVLS grown tetracene single crystal field effect transistors. 

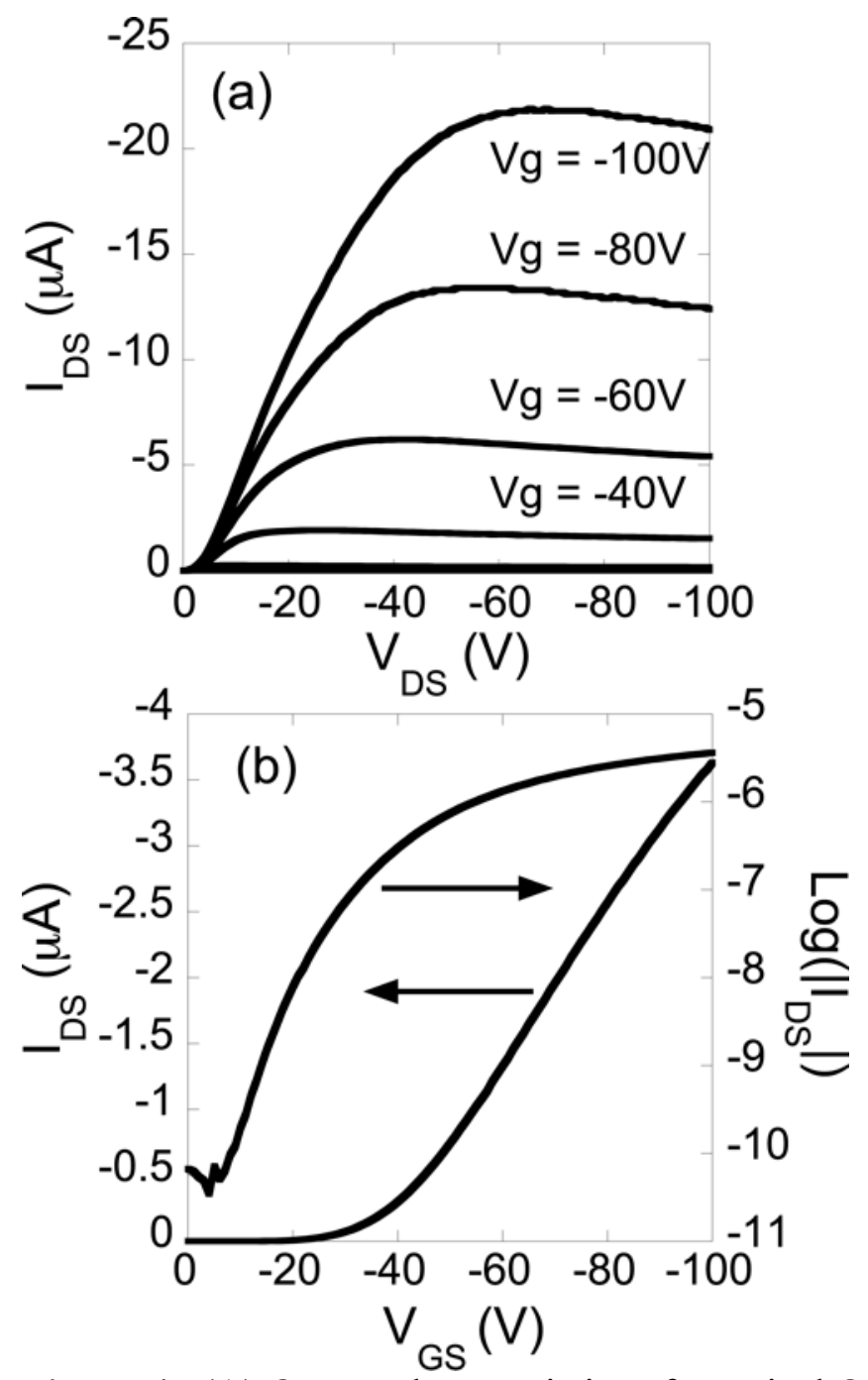

Figure 4. (A) Output characteristics of a typical OVLS grown tetracene single-crystal OFET. (B) Linear transfer characteristics of the same device taken at $V_{S D}=-10 \mathrm{~V}$. 
Graphical Abstract
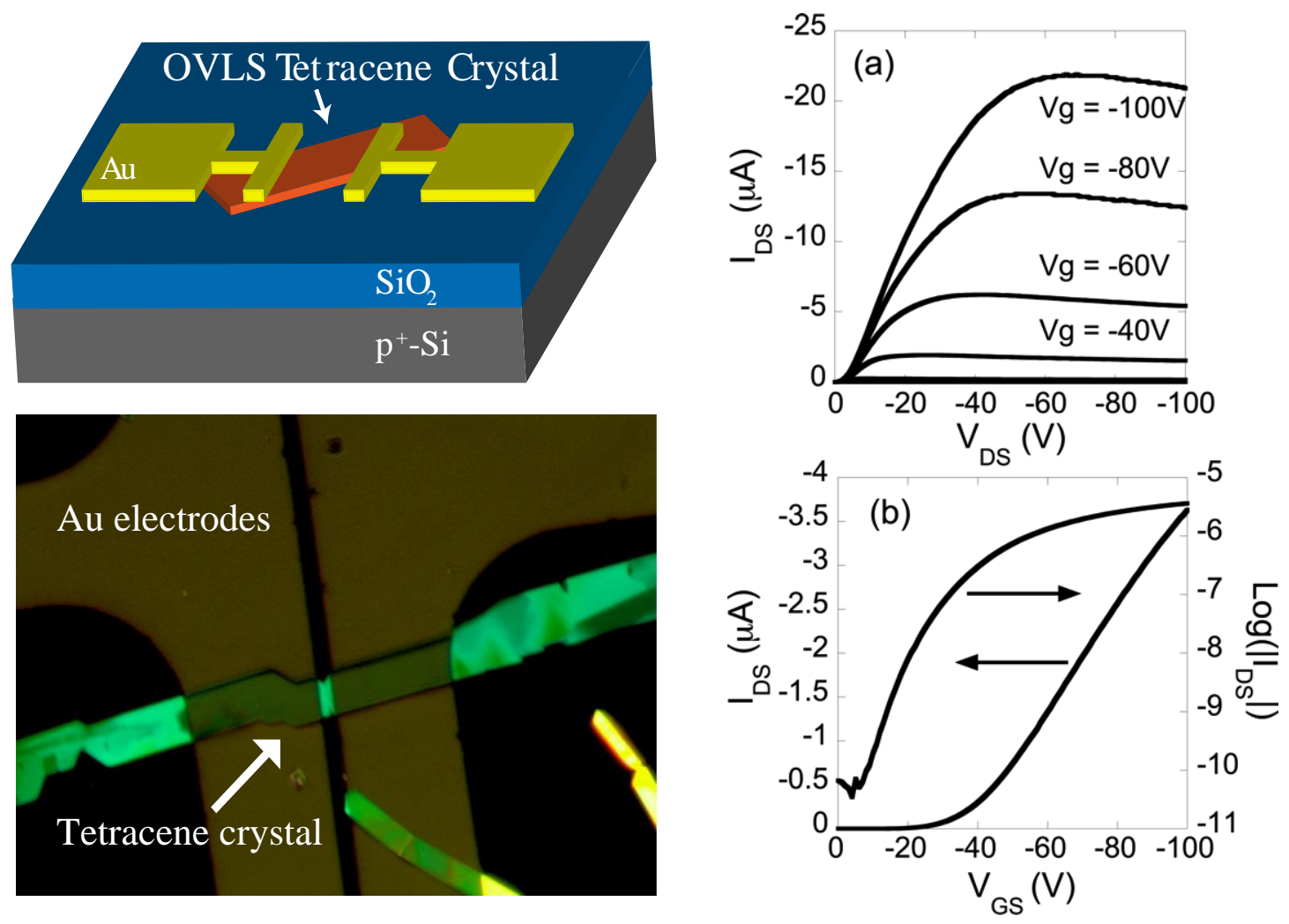